PROCEEDINGS OF THE

AMERICAN MATHEMATICAL SOCIETY

Volume 126, Number 5, May 1998, Pages 1349-1353

S 0002-9939(98)04702-9

\title{
ON THE CHARACTERIZATION OF CERTAIN SIMILARLY ORDERED SUPER-ADDITIVE FUNCTIONALS
}

\author{
HEINZ J. SKALA
}

(Communicated by J. Marshall Ash)

\begin{abstract}
Functionals which behave (sub-, super-) additively on similarly ordered functions occur quite naturally in many contexts. In the present paper we characterize (super-) additive functionals which are defined on a family of functions with the Stone-property in terms of their naturally adjoined dyadic martingales. As corollaries we obtain essential generalizations of integral representations as derived by Schmeidler (1986) and discussed in a recent monograph of Denneberg (1994).
\end{abstract}

\section{INTRODUCTION AND MOTIVATION}

In his systematic study of capacities Choquet [1] associated to increasing set functions $\varphi$ vanishing on the empty set a functional $\Phi$ (often called the Choquet integral with respect to $\varphi$ ) defined by

$$
\begin{aligned}
\Phi(f) & :=\int_{X} f d \varphi:=R \int_{0}^{\infty} \varphi(\{x \in X: f(x) \geq \alpha\}) d \alpha \\
& =: R \int_{0}^{\infty} \varphi(f \geq \alpha) d \alpha, \quad f: X \rightarrow \mathbb{R}^{+},
\end{aligned}
$$

where $R \int_{0}^{\infty}$ denotes the improper Riemann integral.

Dellacherie [2] observed that $\Phi$ as defined above behaves additively on similarly ordered functions.

Definition 1. Two functions $f: X \rightarrow \mathbb{R}$ and $g: X \rightarrow \mathbb{R}$ are said to be similarly ordered, shortly $f$ s.o. $g$, if

$$
(f(x)-f(y))(g(x)-g(y)) \geq 0 \text { for every } x, y \in X .
$$

Let $\mathbb{L}$ denote a family of functions. A functional $\Phi: \mathbb{L} \rightarrow \mathbb{R}$ is said to be s.o.-additive if $\Phi(f+g)=\Phi(f)+\Phi(g)$ whenever $f$ s.o. $g$ and $f+g \in \mathbb{L}$.

Schmeidler [8] called such functionals comonotonic. For historical reasons - the notion similarly ordered has already been used in Hardy, Littlewood, and Pólya ([6], p. 168) - and in order not to confound comonotonic with monotonic in the same direction, we prefer the older more intuitive notion.

It should be noted that s.o.-additive functionals have played a prominent role in statistics since the publications of Dempster, Huber, Shafer and Strassen. They

Received by the editors August 29, 1996.

1991 Mathematics Subject Classification. Primary 28C05; Secondary 47H07, 60A05, 90A05.

Key words and phrases. Similarly ordered, comonotonic, integral representation, martingales.

(C) 1998 American Mathematical Society 
naturally occur in other fields like mathematical economics, game theory and artificial intelligence.

Therefore it seems interesting to characterize them in a uniform way, thus making it possible to apply the sophisticated and well understood methods of measure theory instead of ad hoc considerations. For more background material and a systematic approach to non-additive measures and integrals we refer the reader to Denneberg [3]. The present paper was mainly motivated by the design of a decision theory which is able to handle in a mathematically sound way paradoxical situations like the ones considered by Allais (Nobel Prize 1988) and Ellsberg. Such theories have recently been proposed by Gilboa [4] and Schmeidler [9]. Both theories essentially depend on Schmeidler's [8] representation theorem. The latter result is very special and only applicable if the functional already defines "distribution functions" of the functions on which it is defined. The representation theorem then is simply obtained by integrating the distribution functions. In the following we give characterizations of some classes of s.o.-(super-) additive functionals in terms of the naturally adjoined adapted stochastic process. For this purpose we only need some basic results from the theory of martingales. Our standard reference will be Kopp [7]. As immediate corollaries we obtain essential generalizations of integral representations as discussed by Schmeidler [8]. Note that his ideas are restricted to the case where $\Phi\left(1_{(f \geq \alpha)}\right)$ is defined for all $\alpha \in[\inf f$, $\sup f]$, e.g. if $\mathbb{L}$ consists of measurable functions or if $\mathbb{L}$ is a Stone lattice of functions which is conditionally $\sigma$-completely embedded in $\overline{\mathbb{R}}^{\times}$.

\section{Martingale CHARACTERIZATIONS OF CERTAIN S.O.-(SUPER-) ADDITIVE FUNCTIONALS}

In the following we consider a family of functions $\mathbb{L}$ with the properties and

(i) $f(x) \geq 0$ for all $x \in X$, $\sup _{x \in X} f(x)<+\infty$,

(ii) $\alpha f, f \wedge \alpha, f-f \wedge \alpha \in \mathbb{L}$ if $f \in \mathbb{L}, \alpha \in \mathbb{R}_{+}$.

For a functional $\Phi: \mathbb{L} \rightarrow \mathbb{R}_{+}$the following properties will be of interest:

(iii) $\Phi(\alpha f)=\alpha \Phi(f), f \in \mathbb{L}, \alpha \in \mathbb{R}_{+}$.

(iv) $\Phi(f+g) \geq \Phi(f)+\Phi(g)$, if $f$ s.o. $g$ and $f+g \in \mathbb{L}$.

(v) Let $I_{k}$ be a subset of $\left\{1,2, \ldots, 2^{n_{k}}\right\}$ and let $\left|I_{k}\right| \frac{1}{2^{n_{k}}} \rightarrow 0$ as $k \rightarrow \infty$. Then

$$
\sum_{i \in I_{k}} \Phi\left(f \wedge \frac{i}{2^{n_{k}}}-f \wedge \frac{i-1}{2^{n_{k}}}\right) \rightarrow 0
$$

(vi) If $\left\|f_{n}-f\right\|_{\infty} \rightarrow 0$ as $n \rightarrow \infty$, then $\Phi\left(f_{n}\right) \rightarrow \Phi(f)$.

(vii) $\Phi(f \wedge \alpha) \rightarrow 0$ as $\alpha \rightarrow 0$.

Remarks. 1. For notational convenience we always assume from now on that $\sup f=1$.

2. We state our results for s.o.-superadditive functionals. Corresponding results for s.o.-subadditive functionals are obtained in the same way.

3. Property (vi) has been assumed by Schmeidler [8] for deriving his representation theorem. Obviously (vi) $\rightarrow(\mathrm{v})$ and for increasing functionals (v) and (vii) are equivalent. 
We define an increasing sequence $\left(\mathbb{F}_{n}\right)_{n \in \mathbb{N}}$ contained in the Lebesgue probability space $([0,1], \mathbb{B}, \mu)$ by

$$
\mathbb{F}_{0}:=\{\phi,[0,1]\}
$$

and

$$
\mathbb{F}_{n}:=\sigma \text {-field generated by the atoms }\left[0, \frac{1}{2^{n}}\right), \ldots,\left[\frac{2^{n}-1}{2^{n}}, 1\right] .
$$

Let $\mathbb{F}:=\sigma\left(\bigcup_{n \in \mathbb{N}} \mathbb{F}_{n}\right)$. It is easy to see that in this case the conditional expectations of an integrable random variable $\varphi$ are obtained by

$$
\begin{aligned}
& E\left(\varphi \mid \mathbb{F}_{0}\right)=\left(\int_{0}^{1} \varphi d \mu\right) \cdot 1_{[0,1]}, \\
& E\left(\varphi \mid \mathbb{F}_{n}\right)=\sum_{i=1}^{2^{n}} 2^{n}\left(\int_{(i-1) / 2^{n}}^{i / 2^{n}} \varphi d \mu\right) 1_{\left[\frac{i-1}{2^{n}}, \frac{i}{2^{n}}\right)} .
\end{aligned}
$$

Lemma 1. Let $(\mathbb{L}, \Phi)$ satisfy (i)-(iv). To every $f \in \mathbb{L}$ there is naturally adjoined a positive dyadic supermartingale $\varphi^{f}=\left(\varphi_{n}^{f}, \mathbb{F}_{n}\right)_{n \in \mathbb{N}}$ which, in general, decomposes uniquely into three non-trivial components such that $\varphi^{f}=\varphi^{f 1}+\varphi^{f 2}+\varphi^{f 3}$ where $\varphi^{f 1}$ is a uniformly integrable martingale, $\varphi^{f 2}$ is a martingale converging pointwise a.s. to 0 and $\varphi^{f 3}$ is a potential.

Proof. Define $\varphi_{0}^{f}(\alpha):=\Phi(f) 1_{[0,1]}($ remember $\sup f=1$ !),

$$
\varphi_{n}^{f}(\alpha):=\sum_{i=1}^{2^{n}} 2^{n} \Phi\left(f \wedge \frac{i}{2^{n}}-f \wedge \frac{i-1}{2^{n}}\right) 1_{\left[\frac{i-1}{2^{n}}, \frac{i}{2^{n}}\right)} .
$$

We observe that for any $f \in \mathbb{L}$ the partial sums of functions $f \wedge \frac{i}{2^{n}}-f \wedge \frac{i-1}{2^{n}} \in \mathbb{L}$ are s.o. and

$$
\begin{aligned}
E\left(\varphi_{n+1}^{f} \mid \mathbb{F}_{n}\right)=\sum_{i=1}^{2^{n}} 2^{n}\left[\Phi \left(f \wedge \frac{2 i}{2^{n+1}}\right.\right. & \left.-f \wedge \frac{2 i-1}{2^{n+1}}\right) \\
& \left.+\Phi\left(f \wedge \frac{2 i-1}{2^{n+1}}-f \wedge \frac{2 i-2}{2^{n+1}}\right)\right] 1_{\left[\frac{i-1}{2^{n}}, \frac{i}{2^{n}}\right)} .
\end{aligned}
$$

By (iv)

$$
\begin{aligned}
\Phi\left(f \wedge \frac{i}{2^{n}}-f \wedge \frac{i-1}{2^{n}}\right) \geq & \Phi\left(f \wedge \frac{2 i}{2^{n+1}}-f \wedge \frac{2 i-1}{2^{n+1}}\right) \\
& +\Phi\left(f \wedge \frac{2 i-1}{2^{n+1}}-f \wedge \frac{2 i-2}{2^{n+1}}\right)
\end{aligned}
$$

whence $\varphi_{n}^{f}(\alpha) \geq E\left(\varphi_{n+1}^{f} \mid \mathbb{F}_{n}\right)$ a.s.

As $\varphi$ is a positive supermartingale it converges a.s. to an integrable limit (cf. Kopp [7], p. 51). Denote the a.s. limit of $\left(\varphi_{n}^{f}\right)_{n \in \mathbb{N}}$ by $\varphi_{\infty}^{f}$ and define $\varphi_{n}^{f 1}:=$ $E\left(f_{\infty} \mid \mathbb{F}_{n}\right)$ and $\bar{\varphi}_{n}^{f 2}:=\varphi_{n}^{f}-\varphi_{n}^{f 1}$. Then $\varphi_{n}^{f 1}$ is a uniformly integrable martingale and the supermartingale $\left(\bar{\varphi}_{n}^{f 2}\right)_{n \in \mathbb{N}}$ converges a.s. to 0. We now apply the Riesz decomposition (cf. Kopp [7], p. 68) to $\left(\bar{\varphi}_{n}^{f 2}\right)_{n \in \mathbb{N}}$ to obtain $\varphi^{f 2}$ and $\varphi^{f 3}$.

We are now going to characterize a special class of s.o. superadditive functionals.

Theorem 2. Let $(\mathbb{F}, \Phi)$ satisfy (i)-(iv). If $\Phi$ also satisfies (v), then for every $f \in \mathbb{L}$ the naturally adjoined dyadic supermartingale is uniformly integrable. 
Proof. Assume the contrary. Then for some $f \in \mathbb{L}$ the adjoined supermartingale $\varphi^{f}$ is not uniformly integrable. In the Riesz decomposition $\varphi^{f}=\varphi^{f 1}+\varphi^{f 2}$ the potential $\varphi^{f 2}$ is uniformly integrable, whence the martingale $\varphi^{f 1}$ is not. Equivalently, $\left(\varphi^{f 1}\right)_{n \in \mathbb{N}}$ is not relatively weakly compact in $L^{1}$ (cf. Kopp [7], p. 30). The isometric isomorphism provided by the Radon-Nikodym theorem implies that the sequence $\left(\gamma_{n}\right)_{n \in \mathbb{N}}$ defined by

$$
\gamma_{n}(A):=\int_{A} \varphi_{n}^{f 1} d \mu, \quad A \in \mathbb{B},
$$

cannot be a relatively weakly compact subset of the space of regular Borel measures on $[0,1]$. A celebrated result of Grothendieck [5] tells us that this is equivalent to the existence of an $\varepsilon>0$ and a sequence of disjoint open sets $\left(0_{k}\right)_{k \in \mathbb{N}}$ together with a subsequence $\left(\gamma_{n_{k}}\right)_{k \in \mathbb{N}}$ such that $\gamma_{n_{k}}\left(0_{k}\right)>\varepsilon$ for all $k \in \mathbb{N}$. This in turn gives a subsequence $\left(\varphi_{n_{k}}\right)_{k \in \mathbb{N}}$ such that

$$
\int_{0_{k}} \varphi_{n_{k}}^{f 1} d \mu>\varepsilon \text { for all } k \in \mathbb{N} .
$$

As the set of all $B$ with the property that for every $\delta>0$ there exists a $C \in \bigcup_{n \in \mathbb{N}} \mathbb{F}_{n}$ such that $\mu(B \Delta C)<\varepsilon$ forms a monotone class which contains $\bigcup_{n \in \mathbb{N}} \mathbb{F}_{n}$, a dense subset of the Borel field $\mathbb{B}$, for every $k \in \mathbb{N}$ we can find a $C_{k} \in \mathbb{F}_{n_{k}}$ such that $\varphi_{n_{k}}^{f 1}$ is $\mathbb{F}_{n_{k}}$-measurable and

$$
\left|\int_{C_{k}} \varphi_{n_{k}}^{f 1} d \mu-\int_{0_{k}} \varphi_{n_{k}}^{f 1} d \mu\right| \leq \int_{C_{k} \Delta 0_{k}} \varphi_{n_{k}}^{f 1} d \mu \leq \mu\left(C_{k} \Delta 0_{k}\right) \sup \varphi_{n_{k}}^{f 1}<\frac{1}{2^{n_{k}}} \varepsilon,
$$

whence

$$
\int_{C_{k}} \varphi_{n_{k}}^{f 1} d \mu>\frac{\varepsilon}{2} \quad \text { for all } k \in \mathbb{N} .
$$

Each $C_{k}$ consists of finitely many intervals of length $1 / 2^{n_{k}}$ to which in turn there correspond elements of the form $f \wedge \frac{i}{2^{n_{k}}}-f \wedge \frac{i-1}{2^{n_{k}}} \in \mathbb{L}$.

Observing the s.o.-additivity of the partial sums of such elements we obtain

$$
\begin{aligned}
\frac{\varepsilon}{2} & <\int_{C_{k}} \varphi_{n_{k}}^{f 1} d \mu=\sum_{i \in I_{k}} \Phi\left(f \wedge \frac{i}{2^{n_{k}}}-f \wedge \frac{i-1}{2^{n_{k}}}\right) \\
& =\Phi\left(\sum_{i \in I_{k}}\left(f \wedge \frac{i}{2^{n_{k}}}-f \wedge \frac{i-1}{2^{n_{k}}}\right)\right) \text { for some } I_{k} \subset\left\{1,2, \ldots, 2^{n_{k}}\right\} .
\end{aligned}
$$

As $\left(\sup \varphi_{n_{k}}^{f 1}\right)_{k \in \mathbb{N}}$ cannot be a null-sequence, $\left(\mu\left(C_{k} \Delta 0_{k}\right)\right)_{k \in \mathbb{N}}$ must be one and therefore $\mu\left(C_{k}\right) \rightarrow 0$ as $n \rightarrow \infty$. This gives a contradiction to (v) by observing

$$
\left|I_{k}\right| \frac{1}{2^{n_{k}}}=\mu\left(C_{k}\right)
$$

The following corollaries now easily follow:

Corollary 3. Let $(\mathbb{F}, \Phi)$ satisfy (i)-(iii) and assume that $\Phi$ is s.o.-additive. Then $\Phi(f) \geq \int_{0}^{1=\sup f} \varphi_{\infty}^{f} d \mu$ where $\varphi_{\infty}^{f}$ denotes the closure of the uniformly integrable part of the adjoined dyadic martingale. A proper integral representation, i.e. $\Phi(f)=$ $\int_{0}^{1=\sup f} \varphi_{\infty}^{f} d \mu$, obtains iff $\Phi$ satisfies (v). 
Corollary 4. Let $(\mathbb{F}, \Phi)$ satisfy (i)-(iii) and assume that $\Phi$ is s.o.-additive and increasing. Then $\Phi(f)=R \int_{0}^{1=\sup f} \varphi_{\infty}^{f}(\alpha) d \alpha$ if there exists an auxiliary function $a_{f} \in \mathbb{L}$ such that $f(x) \neq 0 \Rightarrow a_{f}(x) \geq 1$. (Here $R \int$ denotes the proper Riemann integral.)

Remarks. 1. Without the preceding results a proof of Corollary 4 is easily obtained by applying Helley's selection theorem and Arzela's dominated convergence theorem.

2. For increasing functionals $\Phi$ it makes sense to define set functions $\varphi^{\uparrow}$ and $\varphi^{\downarrow}$ as below:

$$
\begin{gathered}
\varphi^{\uparrow}(\{f \geq \alpha\}):=\sup \left\{\Phi(g): g \in \mathbb{L}, g \leq 1_{\{f \geq \alpha\}}\right\}, \\
\varphi^{\downarrow}(\{f \geq \alpha\}):=\inf \left\{\Phi(g): g \in \mathbb{L}, g \geq 1_{\{f \geq \alpha\}}\right\}, \quad \alpha>0 .
\end{gathered}
$$

It is easy to see that $\varphi^{\uparrow}(\{f \geq \alpha\}) \leq \varphi_{\infty}^{f}(\alpha) \leq \varphi^{\downarrow}(\{f \geq \alpha\})$ a.s. and the representation theorems discussed by Denneberg ([3], p. $155 \mathrm{ff})$ may now be inferred from Corollary 4.

If $\Phi$ has further continuity properties (properties (iv) and (v) in Denneberg ([3], p. 156)) we obtain representation results in terms of an improper Riemann integral.

\section{REFERENCES}

1. G. Choquet, Theory of capacities, Ann. Inst. Fourier 5 (1953/54), 131-295. MR 18:295g

2. C. Dellacherie, Quelques commentaires sur les prolongements de capacités, Séminaire de probabilités V, Strasbourg, 77-81, Springer, Berlin, Heidelberg, New York (1971). MR 52:3568

3. D. Denneberg, Non-Additive Measure and Integral, Kluwer Academic Publishers, Doordrecht/Boston/London (1994). MR 96c:28017

4. I. Gilboa, Expected utility with purely subjective non-additive probabilities, J. Math. Ec. 16 (1987), 65-88. MR 88e:90012

5. A. Grothendieck, Sur les applications linéaires faiblement compactes d'espace du type $C(K)$, Canad. J. Math. 5 (1953), 129-173. MR 15:438b

6. G. H. Hardy, J. E. Littlewood and G. Polya, Inequalities, Cambridge Univ. Press, Cambridge (1934).

7. P. E. Kopp, Martingales and Stochastic Integrals, Cambridge Univ. Press, Cambridge (1984). MR 86i: 60004

8. D. Schmeidler, Integral representation without additivity, Proc. A.M.S. 97 (1986), 255-261. MR 87f: 28014

9. D. Schmeidler, Subjective probability and expected utility without additivity, Econometrica $\mathbf{5 7}$ (1989), 571-587. MR 90e:90019

Department of Statistics, University of Paderborn, Warburgerstrasse 100, 33095 PADERBorn, GERMANY 